Research Article

\title{
Network Pharmacology and Metabolomics Studies on Antimigraine Mechanisms of Da Chuan Xiong Fang (DCXF)
}

\author{
Shiyu Ma, ${ }^{1,2}$ Lin Zheng, ${ }^{3}$ Xiao Lin, ${ }^{1,4}$ Yi Feng, ${ }^{4}$ Ming Yang $\mathbb{D}^{5}$ and Lan Shen $\mathbb{D}^{1,4}$ \\ ${ }^{1}$ School of Pharmacy, Shanghai University of Traditional Chinese Medicine, Shanghai, China \\ ${ }^{2}$ Department of Pharmacy, Ruijin Hospital, Affiliated to Shanghai Jiaotong University School of Medicine, Shanghai, China \\ ${ }^{3}$ Department of Bone and Joint Surgery, Shanghai GuangHua Hospital of Integrated Traditional Chinese and Western Medicine, \\ Shanghai, China \\ ${ }^{4}$ Engineering Research Center of Modern Preparation Technology of Traditional Chinese Medicine of the Ministry of Education, \\ Shanghai University of Traditional Chinese Medicine, Shanghai, China \\ ${ }^{5}$ Department of Good Clinical Practice, Longhua Hospital, Affiliated to Shanghai University of Traditional Chinese Medicine, \\ Shanghai, China \\ Correspondence should be addressed to Ming Yang; yangpluszhu@sina.com.cn and Lan Shen; alansusu@sina.com
}

Received 22 November 2020; Revised 1 March 2021; Accepted 5 April 2021; Published 21 April 2021

Academic Editor: Maria Ferraro

Copyright (c) 2021 Shiyu Ma et al. This is an open access article distributed under the Creative Commons Attribution License, which permits unrestricted use, distribution, and reproduction in any medium, provided the original work is properly cited.

\begin{abstract}
Background. Da Chuan Xiong Fang (DCXF) is a traditional Chinese medicine (TCM) formula used to treat migraines. Previously, we uncovered partial mechanisms involved in the therapeutic actions of DCXF on migraines. Methods. In this study, we further elucidated its antimigraine mechanisms in vivo by using an integrated strategy coupling with network pharmacology and metabolomics techniques. Results. Network pharmacology identified 33 genes linked with both migraine and DCXF, most of which were 5-hydroxytryptamine receptors, dopamine, and peptide receptors. The results of GO and KEGG enrichment analysis showed that DCXF significantly regulated tyrosine metabolism, tryptophan metabolism, dopamine metabolic process, glucose transmembrane transport, lipid metabolism, and fatty acid transport. The results of metabolomics analysis found that the metabolism of tryptophan and tyrosine in the brain tissue and energy and lipid metabolism of rats tended towards normal and reached normal levels after administering DCXF. The metabolomics and network pharmacology approaches demonstrated similar antimigraine effects of DCXF on endogenous neurotransmitters and overall trends in serum and brain tissue. Using both approaches, 62 hub genes were identified from the protein-protein interaction (PPI) network of DCXF and gene-metabolite interaction network, with hub genes and different metabolites in serum and brain tissue. The hub genes of DCXF, which were mostly linked with inflammation, might affect mainly neurotransmitters in serum and brain tissue metabolisms. Conclusion. Network pharmacology and metabolomics study may help identify hub genes, metabolites, and possible pathways of disease and treatment. Additionally, two parts of the results were integrated to confirm each other. Their combination may help elucidate the relationship between hub genes and metabolites and provide the further understanding of TCM mechanisms.
\end{abstract}

\section{Background}

Migraine is a recurrent, common debilitating condition with neurovascular pathophysiology. The 2016 Global Burden of Disease study found that it affects an estimated 1.04 billion people (18.9\% of women and $9.8 \%$ of men), with the highest prevalence in Europe and the lowest in Africa [1]. It is the second most disabling condition worldwide and a highly burdensome condition for individuals, families, and society
[2]. It results in a high cost to healthcare insurance systems and patients due to the lost productive time. In mild migraine attacks, medications such as nonsteroidal anti-inflammatory drugs (NSAIDs), for example, acetaminophen and aspirin are used, whereas dihydroergotamine (DHE) or triptans are used for the treatment of moderate-to-severe migraine. Usually, migraine is associated with cardiovascular disease, psychiatric disease, and sleep disorders. However, triptans should not be prescribed to patients with 
a history or risk factors of coronary artery disease [3]. Medication overuse headache $(\mathrm{MOH})$ occurs in predisposed subjects affected by migraine or tension-type headache. In this condition, overuse of drugs such as triptans, for example, for more than 10 days per month over three months, may transform the headache from an episode to a chronic state. $\mathrm{MOH}$ has limited the medication of migraine to a certain degree $[3,4]$.

Da Chuan Xiong Fang (DCXF) was derived from the "Xuanming Lun Fang" (an ancient Chinese medical book written in A. D. 1172), which contained two herbs, Chuan Xiong/LC (Ligusticum striatum DC.) and Tian Ma/GE (Gastrodia elata Blume). It has been extensively applied in treating migraines for thousands of years. We have previously demonstrated that DCXF may decrease blood-brain barrier (BBB) permeability and maintain its integrity through regulating serotonin, excitatory amino acids (EAAs), and matrix metalloprotein-9 (MMP-9) in rats with migraine [5-7]. Meanwhile, DCXF has also played roles in treating migraines through upregulation of 5-HT levels and downregulation of L-Glu levels and calcitonin gene-related peptide (CGRP) expressions in the hypothalamus and periaqueductal gray (PAG), reducing CGRP synthesis and inhibiting neurogenic inflammation [8]. These findings suggest that DCXF may treat migraine by regulating endogenous neurotransmitters. Recent studies showed that DCXF may improve the metabolic profile of serum and trigeminocervical complex (TCC) in rats with migraine through effects on glutamate, aspartic acid, and alanine metabolic processes and also showed the time extent of treatment effects. The possible metabolism pathway of DCXF in urine was mainly kynurenine-tryptophan (TrpKYN) metabolism, and kynurenine was the product of the tryptophan metabolism pathway $[9,10]$.

Network pharmacology, as one of the new technologies to comprehensively and systematically demonstrate the therapeutic mechanisms of TCM, has been successfully and widely applied in many related research fields $[11,12]$. This approach largely reflects the holistic and systematic characteristics of TCM and has clear advantages over the conventional methods in the understanding of illustration of comprehensive mechanisms [11-14]. Metabolomics is an ideal tool for the evaluation the effects of exogenous substances (drugs) on humans by tracking the products of metabolic pathways. In recent years, it has played a critical role in illustrating the possible mechanisms of TCM treatment on diseases such as diabetes and hyperthyroidism [15]. It is a strategy to find active ingredients and validate the effectiveness and mechanism of network pharmacology, confirm the effective active products of the metabolic process, and investigate the prototype compounds [16-20].

In this study, network pharmacology and metabolomics were both used to investigate the neurotransmission and metabolic mechanisms of DCXF for the treatment of migraine. Network pharmacology was applied to discover the possible therapeutic targets from multiple ingredients in LC and GE and illustrate the possible pathways in antimigraine mechanisms. Metabolomics was used to identify the possible metabolic mechanism by which DCXF has an effect on migraines.

\section{Methods}

2.1. Chemicals and Reagents. The two crude drugs Ligusticum striatum DC. (LC) and Gastrodia elata Blume (GE) were purchased from Shanghai Kangqiao Chinese Herbal Medicine Co., Ltd. (Shanghai, China) and were identified by Professor Yajun CUI (Department of Chinese Pharmacy, Shanghai University of Traditional Chinese Medicine) under the guidance of Chinese Pharmacopeia 2020 edition. Voucher specimens (ID: 010-5 tianma-01 and 010-chuanxiong-01) were deposited in the Museum of Shanghai University of Traditional Chinese Medicine, Shanghai University of Traditional Chinese Medicine, China. Active components of both LC and GE were identified in the preliminary studies [16-18]. Pellets were formed from the active components of LC and GE using extrusion-spheronization technology.

DCXF was formulated as follows: component A was formed from LC pellets of 33\% drug loading for active components (including 9.52\% ferulic acid and $18.31 \%$ senkyunolide I); component B was formed from GE pellets of $33 \%$ drug loading for active components (including $6.13 \%$ gastrodin). Components $\mathrm{A}$ and $\mathrm{B}(0.95 \mathrm{~g}$ and $2.15 \mathrm{~g}$, resp.; dose ratio of $4: 9)$ were then dissolved in $10 \mathrm{ml} 0.9 \%$ normal saline (NS). The preparation, quality control, and highperformance liquid chromatography (HPLC) of DCXF, GE, and LC are shown in Supplementary S1.

Methanol, acetonitrile, and chloroform (chromatographic grade) were obtained from Merck Chemicals (Germany). Pyridine, L-thyroxine sodium, methoxyamine, and N, O-bis (trimethylsilyl) trifluoroacetamide (BSTFA) containing $1 \%$ trimethylchlorosilane (TMCS) used in the study were all purchased from Sigma-Aldrich (St. Louis, MO, USA). Nitroglycerin injection was purchased from Beijing Yimin Pharmaceutical Co., Ltd. (China). L-2Chlorophenylalanine at $0.3 \mathrm{mg} / \mathrm{mL}$ in water was prepared and used as the internal standard. Ultrapure water was prepared with a Milli-Q system (Millipore, Billerica, MA, USA). The assay kits for serotonin (5-HT), 5-hydroxy indoleacetic acid (5-HIAA), nitrogen monoxide (NO), nitric oxide synthase (NOs), calcitonin gene-related peptide (CGRP), and dopamine (DA) were all purchased from the Beijing North Biotechnology Institute (Beijing, China).

The standards of ferulic acid, 4-hydroxy benzyl alcohol, and gastrodin were purchased from the National Institute for Food and Drug Control (Beijing, China) with a minimum purity of $98 \%$. The standards of senkyunolide I, senkyunolide $H$, butylphthalide, butylidenephthalide, and vanillin were separated from LC with a minimum purity of at least $98 \%$. Parishin was purchased from TAUTO Biotech Co., Ltd. (Shanghai, China) with a minimum purity of $95 \%$. Phosphate was obtained commercially from China Sinopharm Co., Ltd., part of the Shanghai Chemical Reagent Company (Shanghai, China). Milli-Q Pure water system (for HPLC and ultraperformance liquid chromatography (UPLC)) was purchased from the Millipore Company 
(France). Acetonitrile and methanol, both HPLC grade, were purchased from Merck (Germany).

\subsection{Network Pharmacology Analysis}

2.2.1. Ingredients Preparation from DCXF and Target Prediction [21]. Information about the chemical ingredients of LC and GE in the DCXF was gathered from the following literature and data sources: Herb Ingredients' Targets (HIT database); STITCH database; Chinese Academy of Sciences Chemistry database; Traditional Chinese Medicine Systems Pharmacology (TCMSP) database; Traditional Chinese Medicine Integrated Database (TCMID). We collected 49 and 12 chemical ingredients from LC and GE, respectively (Supplementary S2), and their structures were retrieved from the PubChem database (https://pubchem.ncbi.nlm. nih.gov/). The quantitative estimate of drug-likeness (QED) $[11,12]$ was calculated to prescreen pharmaceutically active compounds in DCXF. Those compounds with QED $>0.3$ were chosen for further target prediction analysis. After filtering redundant information, we obtained 46 ingredients, including 44 and two compounds from LC and GE, respectively. All chemical data were then used as a data source for target prediction (Supplementary S3).

Potential targets of these ingredients were analyzed using the score for a specific target (GS) in accordance with relevant literature [21]. A target interacting with many compounds may be regarded as a core target in the pharmacological effects of the formula. We selected the targets with GS $>0$, resulting in 531 core targets (Supplementary S4).

2.2.2. Molecular Docking [22]. PSOVina (http://cbbio.cis. umac.mo) is a hybrid model that combines particle swarm optimization (PSO) global search and BroydenFletcher-Goldfarb-Shannon (BFGS) local search methods in AutoDock Vina to tackle the conformational search problem in docking. It also has the advantage of execution time reduction (by 51-60\%) [22], without compromising the prediction accuracies in the docking and virtual screening experiments. In this docking assay, six human receptors were retrieved from Protein Data Bank (http://www.wwpdb. org/), including human NO (PDB ID : 4N8T), human calcitonin gene-related peptide (PDB ID:3AQF), human dopamine $\mathrm{D}_{3}$ receptor (PDB ID : $3 \mathrm{PBL}$ ), human NOs (PDB ID : 1NSI), human 5-HT1B (PDB ID:4IAR), and human 5HT2AR (PDB ID:6A93). To evaluate the accuracy of the ligand docked pose, the predicted position of each ligand atom was compared with its standard ligand using the standard root-mean-square deviation (RMSD).

\subsubsection{Enrichment Analysis and Network Construction. To} investigate potential pathways regulated by DCXF, enrichment analysis was carried out to identify the significant biological profile of DCXF. The hypergeometric $P$ value has been widely used to investigate genes from predefined functional terms. In this work, gene ontology (GO) enrichment analysis for molecular function (MF), cellular component (CC), and biological process (BP) was performed, and pathway enrichment analysis was based on the Kyoto Encyclopedia of Genes and Genomes (KEGG) pathway database [21].

Analysis of the functional interactions between proteins may provide insights into the mechanisms of DCXF, and in this study, the protein-protein interaction (PPI) network was predicted using the Search Tool for the Retrieval of Interacting Genes (STRING; http://string-db.org) (version 11.0) online database. A combined score $>0.4$ was considered statistically significant. The plug-in application Molecular Complex Detection (MCODE) (version 1.4.2) of Cytoscape (version 3.7.0, https://cytoscape.org/) was an application for clustering a given network based on its topology to find densely connected regions [21]. The PPI networks were drawn using Cytoscape, and the significant modules in the PPI networks were identified using MCODE. The criteria for selection were as follows: MCODE scores $>5$, degree cut-off $=2$, node score cutoff $=0.2$, max. depth $=100$, and k-score $=2$. Subsequently, the KEGG and GO enrichment analyses were performed for genes in these modules. Hub genes were those with degrees $\geq 10$.

Some of the aforementioned analysis was conducted using the Traditional Chinese Medicine Network Pharmacology Analysis System (TCMNPAS). The National Computer Software registration number was 2019SR1127090 (produced by author MY).

2.3. Disease-Associated Genes. In this work, migraine was considered a disease, and 72 associated genes were collected from the GeneCards database [23] (Supplementary S5).

2.4. Animals and Treatment. Twenty-four male SpragueDawley (SD) rats (age: four weeks; body weight: $150 \pm 20 \mathrm{~g}$ ) were supplied by the Lab Animal Center of Shanghai University of Traditional Chinese Medicine (SCXK 20130016). For one week prior to experiments, rats were raised in a humidity and temperature-controlled specific pathogenfree room (humidity: $60 \%$, temperature: $22 \pm 3^{\circ} \mathrm{C}$ ) in the Experimental Animal Center of Shanghai Traditional Chinese Medicine University (Shanghai, China), with a $12 \mathrm{~h}$ light and dark cycle and with free access to food and sterile water. The animal facilities and protocols were approved by the Institutional Animal Care and Use Committee, Shanghai University of Traditional Chinese Medicine, on 4th of September 2016 (no. SZY201609010). All procedures were conducted in accordance with the Guide for the Care and Use of Laboratory Animal (The National Academies Press, revised edition 2010).

After three days, the animals were divided at random into three groups with eight rats in each, control group, nitroglycerin- (NTG-) induced migraine group, and DCXF group, and subjected to the following treatment schedules:

(1) control group $(n=8)$ : NTG vehicle, $10 \mathrm{ml} / \mathrm{kg}$, i.h. + saline $(30 \mathrm{~min})$ 
(2) NTG-induced migraine model group $(n=8)$ : NTG, $10 \mathrm{mg} / \mathrm{kg}$, i.h. + saline $(30 \mathrm{~min})$

(3) $\mathrm{DXCF}+\mathrm{NTG}$ group $(n=8): \quad \mathrm{NTG}, 10 \mathrm{mg} / \mathrm{kg}$, i.h.+DCXF (30 min)

We used the NTG model followed by [24]. NTG was administered subcutaneously at a dosage of $10 \mathrm{mg} / \mathrm{Kg}$. In the DXCF group, physiological saline and DCXF were orally administered 30 minutes after NTG at a dosage of $10 \mathrm{~mL} / \mathrm{Kg}$ (weight). All rats were anesthetized 30 minutes after drug or vehicle administration.

Animal sacrifice involved anesthesia by intraperitoneal injection of ketamine $(100 \mathrm{mg} / \mathrm{Kg})$ and xylazine $(12 \mathrm{mg} / \mathrm{Kg})$ over a period of one hour. Following this, blood was drawn from the abdominal aorta, and each blood sample was centrifuged at $3500 \mathrm{rpm}$ for $15 \mathrm{~min}$ at $4^{\circ} \mathrm{C}$. Brains were removed immediately after collecting blood, and serum and brain tissue samples were stored at $-80^{\circ} \mathrm{C}$.

The following parameters were assessed from the blood samples: NO, NOs, 5-HT, 5-HIAA, DA, and CGRP values. The metabolites of serum and brain tissue were assessed from blood and brain samples, respectively. More details about the animals and treatment are shown in Supplementary S6.

2.5. Biomedical Analysis. Analysis was conducted according to the kit instructions for NO, NOs, 5-HT, 5-HIAA, DA, and CGRP. First, the standard was diluted at five grades, with each gradient sample volume being $50 \mu \mathrm{L}$. The sample dilution of $40 \mu \mathrm{L}$ was added to the sample holes, and then, the testing sample $10 \mu \mathrm{L}$ was added to the holes. After sealing the plate with a membrane, the plate was incubated at $37^{\circ} \mathrm{C}$ for $30 \mathrm{~min}$, after which the membrane was removed, the liquid discarded, and the sample was dried. Each hole was filled with washing liquid, allowed to stand for $30 \mathrm{~s}$, and then emptied, and this process was repeated five times, after which the sample was dried again. Enzyme reagent $(50 \mu \mathrm{L})$ was added to each sample, and again, the samples were membrane-sealed and incubated at $37^{\circ} \mathrm{C}$ for $30 \mathrm{~min}$. After this, the washing cycles previously described were repeated and the samples were dried. The chromogenic reagent $\mathrm{A}$ $(50 \mu \mathrm{L})$ was added and then chromogenic reagent $\mathrm{B}(50 \mu \mathrm{L})$ was added, gently mixed by shaking, and stored in a dark environment at $37^{\circ} \mathrm{C}$ for $15 \mathrm{~min}$. Stop buffer $(50 \mu \mathrm{L})$ was then added to each sample, the reaction was terminated, and within $15 \mathrm{~min}$ of this step, spectrophotometer was set to a baseline of zero, and $450 \mathrm{~nm}$ wavelength sequentially measured the absorbance of each hold (OD).

Data were expressed as means $\pm \mathrm{SD}$. For the comparisons of different groups, one-way analysis of variance (ANOVA) with LSD-t correction for multiple comparisons was conducted using SPSS version 25.0. A $P$ value below 0.05 was taken to indicate a significant difference between data means.

\subsection{Metabolites of Serum and Brain Tissue [15, 25, 26]}

2.6.1. Sample Preparation of Serum. Thawed serum samples were centrifuged at $3500 \mathrm{rpm}$ for $10 \mathrm{~min}$ at $4^{\circ} \mathrm{C}$, and $100 \mu \mathrm{L}$ supernatant was extracted. Methanol $(400 \mu \mathrm{L})$ was added to the supernatant and vortex-mixed for $2 \mathrm{~min}$, and then the mixture was centrifuged at $10,000 \mathrm{rpm}$ for $15 \mathrm{~min}$ at $4^{\circ} \mathrm{C}$. Supernatant $(20 \mu \mathrm{L})$ was transferred into injection vials and kept at $-20^{\circ} \mathrm{C}$ for UPLC-TOF/MS analysis.

2.6.2. Sample Preparation of Brain Tissue. Methanol-water $(200 \mu \mathrm{L})$ solution was added to $50 \mu \mathrm{g}$ brain tissue sample to extract metabolites, and then, the mixture was vigorously vortexed and centrifuged at $12000 \mathrm{rpm}$ for $15 \mathrm{~min}$ at $4^{\circ} \mathrm{C}$. The supernatant was collected and the residues were extracted using $200 \mu \mathrm{L}$ methanol. The resulting aliquots were then mixed with water-extracted supernatants. The mixtures were dried completely and combined with $80 \mu \mathrm{L}$ methoxylamine $(15 \mathrm{mg} / \mathrm{mL}$ in pyridine $)$ at $37^{\circ} \mathrm{C}$ for $2 \mathrm{~h}$, to allow methoxylation. Subsequently, the sample was trimethylsilylated with BSTFA (with $1 \%$ TMCS) at $70^{\circ} \mathrm{C}$ for $1 \mathrm{~h}$. All the processed samples were immediately analyzed using gas chromatography time-of-flight mass spectrometry (GC TOF/MS).

In the GC TOF/MS analysis, we followed the methods of Zhu et al., 2014 [26], to study the metabolites of brain tissue. More details of UPLC- TOF/MS and GC TOF/MS analyses were shown in Supplementary S7

\section{Results}

3.1. Target Prediction and Network Analysis of the Antimigraine Mechanisms of DCXF. Migraine-related genes (72 gene symbols) and core targets of DCXF (531 gene symbols) were identified. Of these, 33 genes were associated with both migraine and DCXF (Table 1). Most of the intersecting genes were 5-hydroxytryptamine receptor (including HTR1B, HTR2A, HTR7, HTR2C, HTR2B, HTR1F, and HTR1A), dopamine receptor (including DRD2, DRD5, DRD3, and DRD4), and peptide receptor (including CALCA, TAC1, HCRT, VIP, NPY, ADCYAP1, and NPS). Overall, these results are consistent with those of our previous studies.

Molecular docking analysis of all active ingredients (including 46 compounds from network pharmacology analysis and seven compounds from HPLC quality analysis) showed that most of the key active ingredients, including validated gastrodin and ferulic acid, were well-docked to proteins of six human receptors, with several interactions with amino acid residues (Table 2 and Supplementary S8). A 3D molecular docking model of the gastrodin and ferulic acid with six proteins is shown in Figure 1.

Further, potential pathways in the pathogenesis of migraine under DCXF treatment were screened out from candidate targets from GO and KEGG enrichment analysis. Figure 2 showed the top $10 \mathrm{GO}$ terms and KEGG pathways associated with DCXF and migraine, respectively. The number of GO (MF and BP) and KEGG terms that were significantly associated with the DCXF targets were 150, 1836, and 141 (adjusted $P$ value $<0.01$ ), respectively. For MF ontology, some of the top GO terms (GO:0030594, GO: 0051378, GO:0004993, GO:0099589, GO:0099528, GO: 0035240, and GO:0042165) associated with migraine were 
TABLE 1: Genes associated with both migraine and DCXF.

Disease gene symbol (33 genes)

CALCA, TNF, HTR1B, TAC1, HTR2A, HTR7, DRD2, HTR2C, MAOA, HTR2B, HTR1F, TACR1, HCRT, HRH3, HTR1A, TRPV1, VIP, NPY, DRD5, SLC6A4, ADCYAP1, NPS, DRD3, DRD4, PPBP, FOS, CNR1, COMT, EDNRA, TRPA1, EDNRB, MAOB, and CYP2D6

TABLE 2: GO and KEGG terms enrichment analysis of DCXF in the most significant module. FDR: false discovery rate.

\begin{tabular}{lccr}
\hline Pathway ID & Pathway description & \% associated gene & FDR \\
\hline GO:0004016 & Adenylate cyclase activity & 72.73 & $1.29^{*} 10^{-21}$ \\
GO:1904321 & Response to forskolin & 60.00 & $1.25^{*} 10^{-15}$ \\
GO:1904322 & Cellular response to forskolin & 60.00 & $1.25^{*} 10^{-15}$ \\
GO:0004383 & Guanylate cyclase activity & 42.11 & $5.86^{*} 10^{-19}$ \\
GO:0008074 & Guanylate cyclase complex, soluble & 42.11 & $5.86^{*} 10^{-19}$ \\
GO:0016849 & Phosphorus-oxygen lyase activity & 34.78 & $3.79^{*} 10^{-18}$ \\
\hline
\end{tabular}

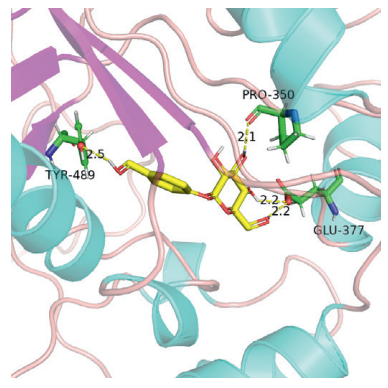

(a)

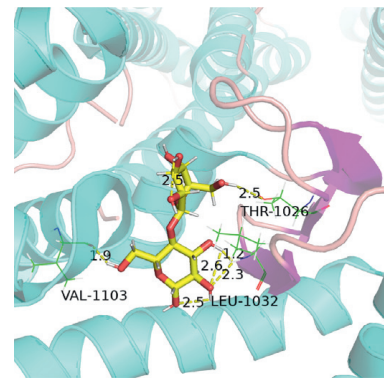

(e)

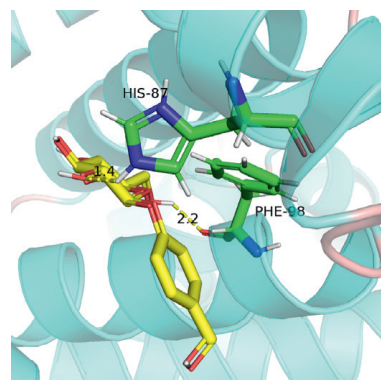

(i)

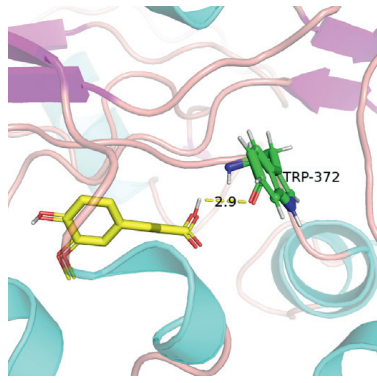

(b)

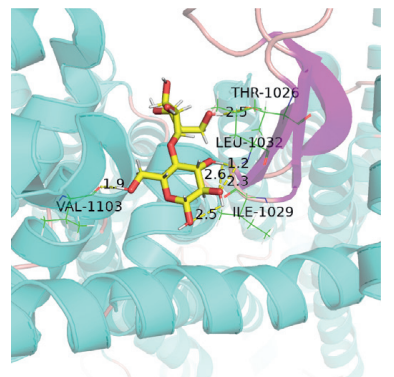

(f)

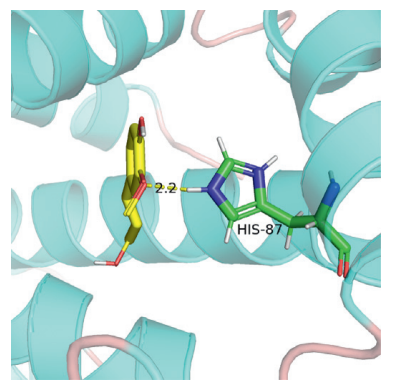

(j)

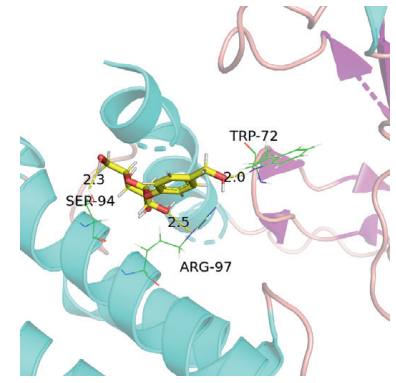

(c)

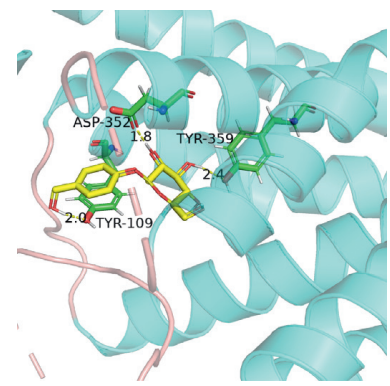

(g)

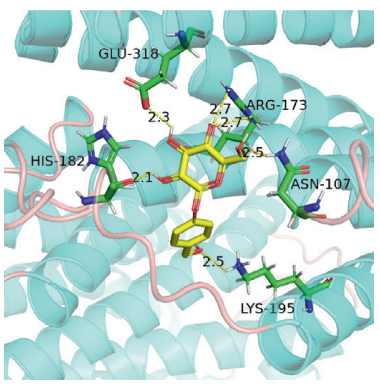

(k)

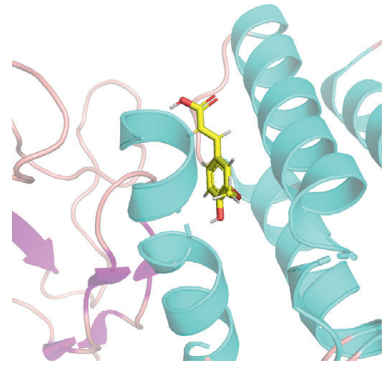

(d)

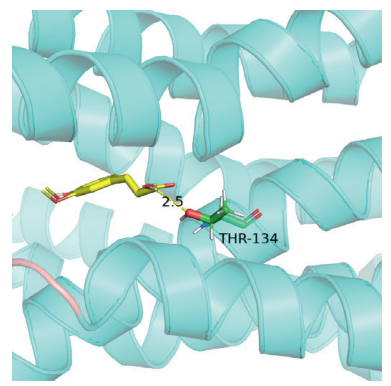

(h)

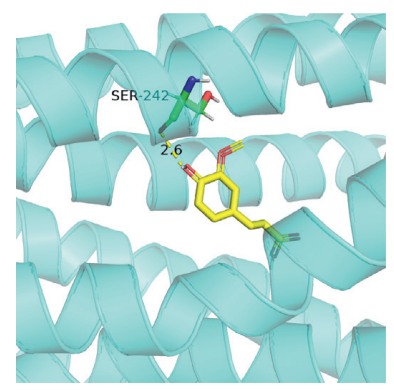

(1)

FIGURE 1: 3D molecular docking model of the gastrodin and ferulic acid with six proteins. (a) Gastrodin and NOs; (b) ferulic acid and NOs; (c) gastrodin and CGRP; (d) ferulic acid and CGRP; (e) gastrodin and dopamine D3; (f) ferulic acid and dopamine D3; (g) gastrodin and 5HT1B; (h) ferulic acid and 5-HT1B; (i) gastrodin and NO; (j) ferulic acid and NO; (k) gastrodin and 5-HT2AR; (l) ferulic acid and 5-HT2AR.

neurotransmitter receptor activity, serotonin binding, G-protein coupled serotonin receptor activity, serotonin receptor activity, G-protein coupled neurotransmitter receptor activity, dopamine binding, and neurotransmitter binding. For BP ontology, there were seven GO terms (GO:
0007187, GO:0008015, GO:0003013, GO:1904659, GO: 0007210, GO:0006836, and GO:0007188), which were G-protein coupled receptor signaling pathway, coupled to cyclic nucleotide second messenger, blood circulation, circulatory system process, glucose transmembrane transport, 
serotonin receptor signaling pathway, and neurotransmitter transport. All were significantly associated with migraine and DCXF $(P<0.05)$. From the KEGG enrichment analysis, ten KEGG pathways (hsa00350, hsa00380, hsa01522, hsa04020, hsa04024, hsa04080, hsa04540, hsa04726, hsa04728, and hsa04750), namely, tyrosine metabolism, tryptophan metabolism, endocrine resistance, calcium signaling pathway, cAMP signaling pathway, neuroactive ligand-receptor interaction, gap junction, serotonergic synapse, dopaminergic synapse, and inflammatory mediator regulation of TRP channels, were associated with migraine and DCXF.

Apart from some well-known migraine-related pathways involved in neurotransmitter receptor activity (peptide receptor activity, serotonin receptor signaling pathway, and calcium signaling pathway), the main finding was that DCXF significantly altered the regulation of the dopamine metabolic process, glucose transmembrane transport, lipid metabolism, fatty acid transport, tyrosine metabolism, and tryptophan metabolism.

Furthermore, the cumulative distribution of the percentages of common enriched-GO pathways was used as a measure for comparing the degree of association between migraine and DCXF [21]. This method was used to gauge the level of probability of association between DCXF and migraine. The significantly enriched-GO terms were combined and sorted in ascending order according to adjusted $P$ values, and the percentage of common terms from the top 30 enriched terms in DCXF for migraine were presented in Figure 2. The area under the cumulative curve (AUC) was calculated using trapezoidal integration. The similarity between DCXF targets and disease genes was 0.79 , and the AUC value was 17.1 for migraine. The results showed a strong association between DCXF targets and migraine genes in terms of GO.

3.2. PPI Network Construction and Module Analysis. The PPI network of DCXF was constructed (number of nodes: 531; number of edges: 11115) and the three significant modules (A: scores 15.143, 29 nodes, and 212 edges; B: scores 6.33, 7 nodes and 19 edges; C: scores 4.727, 12 nodes, and 26 edges) were obtained using Cytoscape MCODE (Figure 3). A total of 62 genes were identified as hub genes with degrees $\geq 10$ (Table 3).

The results of functional analyses showed that genes in three modules were mainly enriched and shown in Supplementary S9 $($ FDR $<0.05)$. Genes in module A were mainly enriched adenylate cyclase activity, guanylate cyclase activity, and response to forskolin. Genes in both modules B and $\mathrm{C}$ were enriched in long-chain fatty acid metabolism, peroxisome proliferator-activated receptor (PPAR) signaling pathway, and cholesterol metabolism.

\subsection{Nitroglycerin (NTG) Modeling and Biomedical Analysis.} The rats from the NTG model group and DCXF group displayed more impatience and anxiety (scratching, crawling, and biting) than the rats in the control group. After administration of DCXF, the rats calmed down and this spontaneous activity reduced.

Plasma levels of 5-HT, 5-HIAA, CGRP, DA, NO, and NOs in the model group were significantly higher than in the controls and in the DCXF group $(P<0.01)$ as shown in Figure 4. This indicates that the migraine model induced by nitroglycerin was successful and that DCXF may have therapeutic effects on the migraine model.

3.4. Effect of DCXF on Serum Metabolic Profiling. A partial least-squares discriminant analysis (PLS-DA) model (Figure 5) was constructed to visualize the influence of DCXF on the metabolic profiling of different treatment groups. This analysis showed clear separations of the control, NTGinduced migraine model, and DCXF groups, suggesting that biochemical changes differed between them (Figure 5). The DCXF profile was closer to that of the control than the model group, indicating that the metabolites had trended towards normal after DCXF injection. Differential metabolites that were accountable for intergroup variation with VIP value $>1.0$ and Student's $t$ test $P$ value $<0.05$ were listed in Supplementary S10 (the significance level was set at $0.05)$.

After searching the HMDB and KEGG databases, the remaining metabolites were identified. Eighteen metabolites were chosen as potential biomarkers: 14 positive modes and four negative modes.

Tryptophan (Trp), tyrosine (Tyr), choline, and phosphatidylcholines (PC) were all decreased in the model group than in controls. Conversely, 5-HT, 5-hydroxytryptophan (5-HTP), 5-HIAA, DA, $\gamma$-aminobutyric acid (GABA), lactic acid, pyruvic acid, valine, (R)-3-hydroxybutyric acid, glutamic acid (Glu), glutamine (Gln), aspartic acid (Asp), and vanylglycol (MHPG) were all increased in the NTG-induced migraine model group compared with controls. On the whole, after DCXF treatment, these metabolites tended to decrease in comparison with the NTG-induced migraine group. Of note, MHPG, GABA, DA, 5-HT, 5-HTP, 5-HIAA, (R)-3-hydroxybutyric acid, lactic acid, and pyruvic acid returned to normal levels after DCXF administration.

\subsection{Effects of DCXF on Brain Tissue Metabolic Profiling.} The PLS-DA model (Figure 5) was constructed to visualize the influence of DCXF on the metabolic profiling of NTGinduced migraine model group rats and identify potential metabolites.

Compared with the control group, arachidonic acid (AA), valine, proline, serine, isoleucine, leucine, Asp, asparagine, Glu, and $\alpha$-alanine increased in the NTG-induced migraine group, while Trp, Tyr, inosine, ribonic acid, and D-ribofuranose decreased in NTG-induced migraine model group.

On the whole, after DCXF treatment, proline, asparagine, Glu, Asp, serine, $\alpha$-alanine, and valine tended closer to normal levels but they did not all reach normal levels. Fifteen metabolites were chosen as potential biomarkers and were listed in Supplementary S10. 


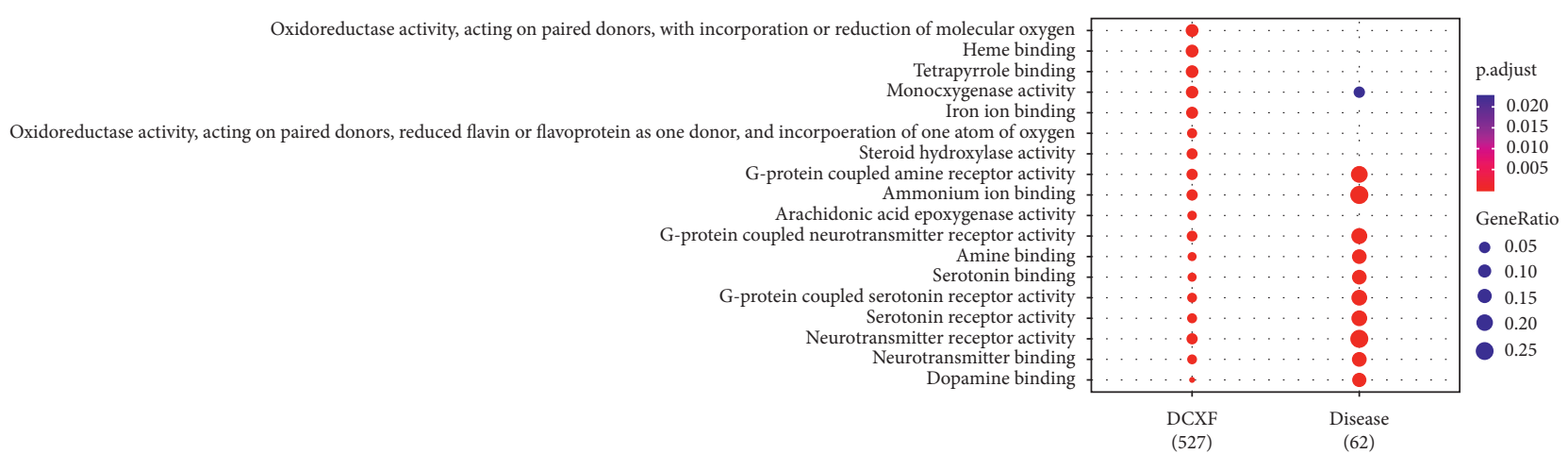

(a)

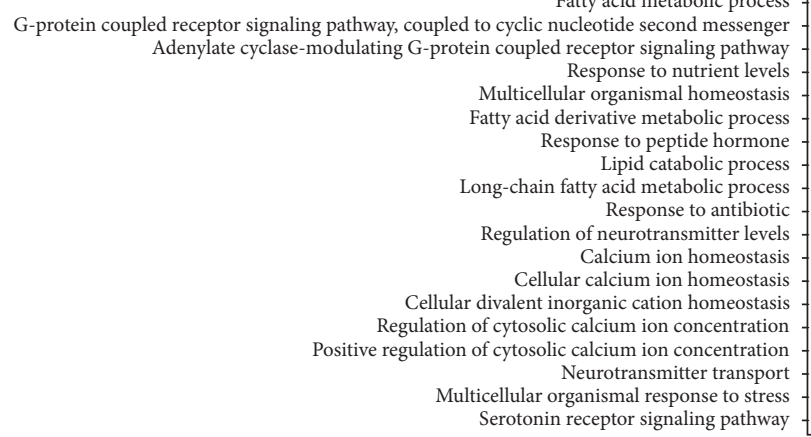
Serotonin receptor signaling pathway
Sellular organismal response to stress

(b)

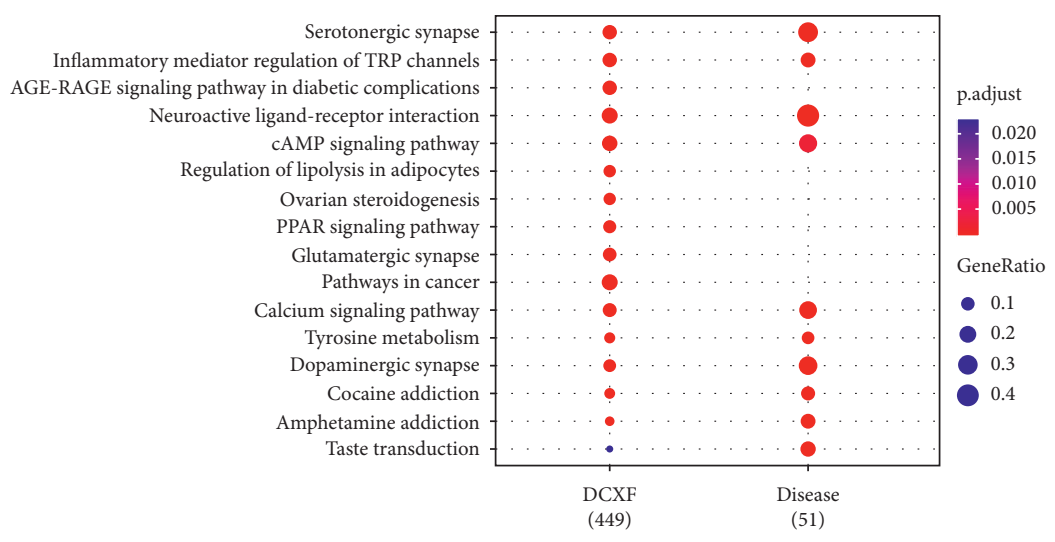

(c)

FIgURe 2: Continued. 


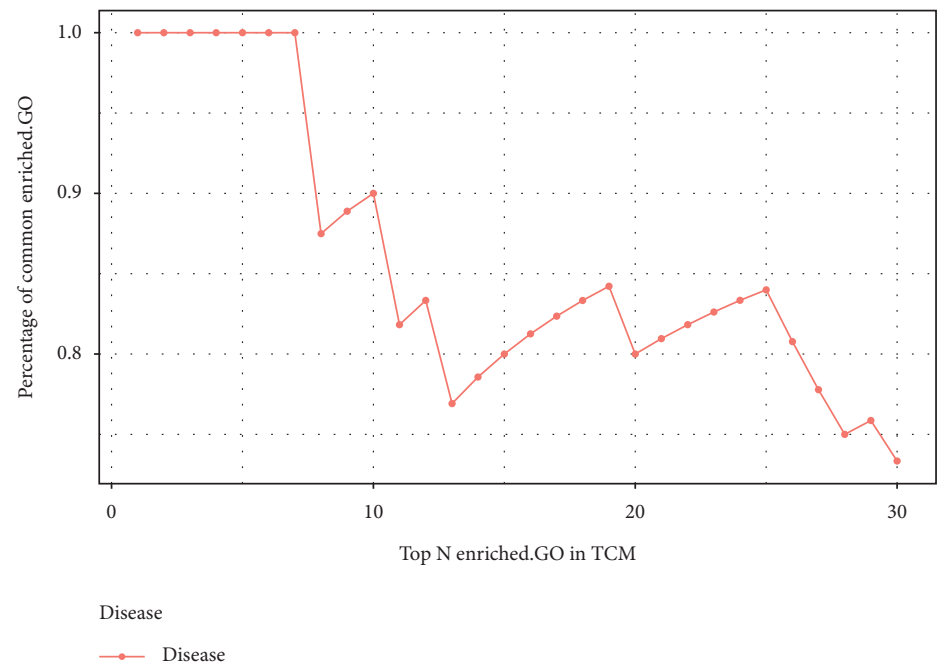

(d)

Figure 2: Top 10 GO terms: (a) MF, (b) BP, (c) KEGG pathways associated with DCXF and migraine, and (d) the cumulative distribution of percentages of common terms from top (30) enriched-GO. The terms are presented in descending $P$ adjust value.

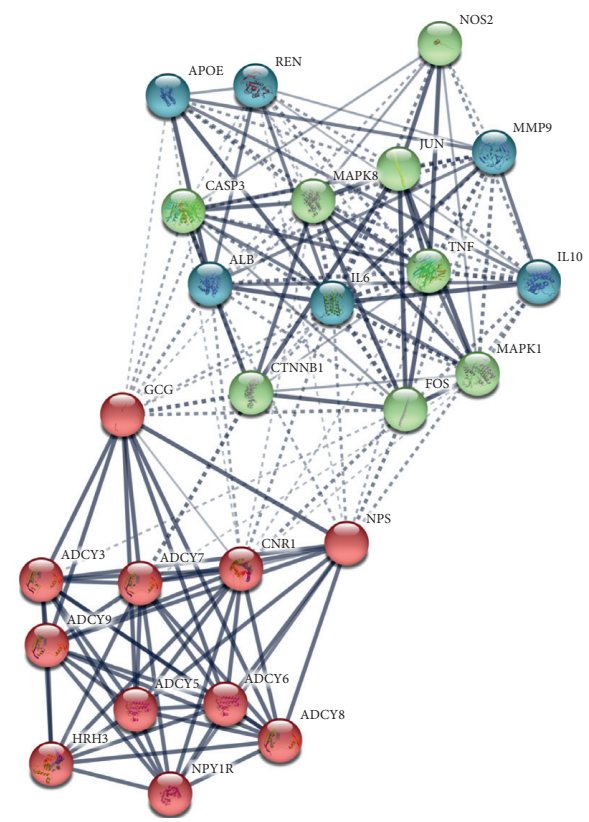

(a)

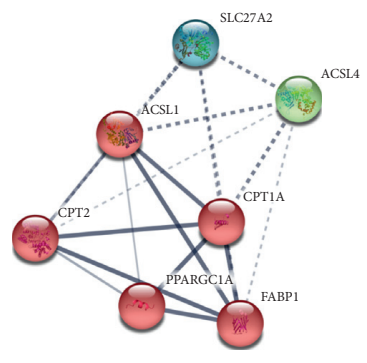

(b)

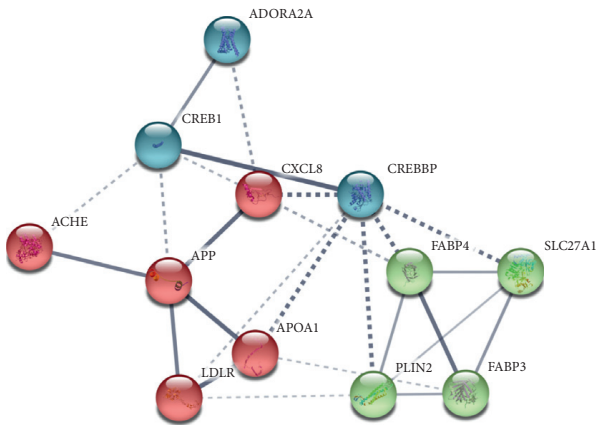

(c)

Figure 3: The PPI network of DCXF.

TABLE 3: Hub genes of DCXF.

Hub genes (62 genes)

MAPK8, JUN, APOA1, APOB, FOS, CREB1, CREBBP, IL10, TNF, IL6, MAPK1, LDLR, APOE, CXCL8, APP, PPARA, PPARGC1A, ALB, FABP1, NPS, HCRT, NOS2, CTNNB1, CASP3, GCG, GNG2, CPT1A, FABP4, CPT2, SLC27A1, PTGS2, ACSL1, PLIN2, ADCY2, ADCY1, ADCY5, ADCY7, MMP9, ADCY8, ADCY9, ADCY6, CHAT, ACHE, COMT, ADCY3, CNR1, HRH3, NPY1R, SLC27A2, FABP3, ADORA2A, and REN

3.6. Possible Pathways. To explore the possible metabolic pathways involved in the therapeutic mechanism, the metabolites that contributed to the change of the metabolic state resulting from DCXF were imported to the MetaboAnalyst 4.0 (https://www.metaboanalyst.ca/home.xhtml) (shown in Table 4). MetaboAnalyst is a comprehensive and 


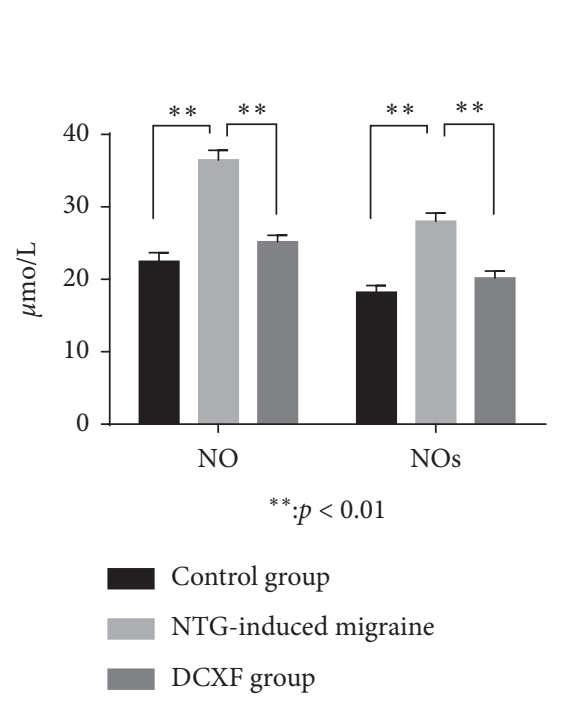

(a)

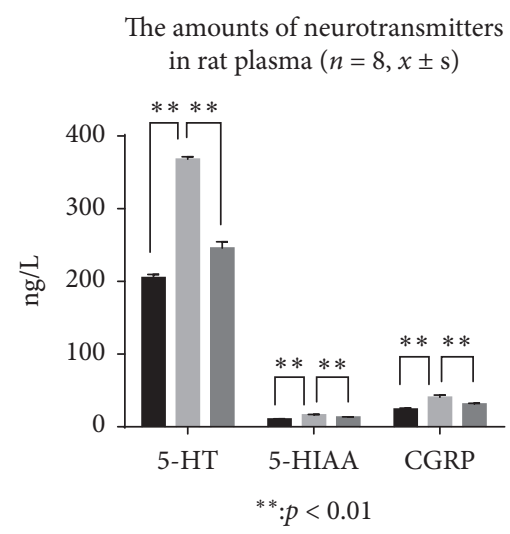

Control group

NTG-induced migraine

DCXF group

(b)

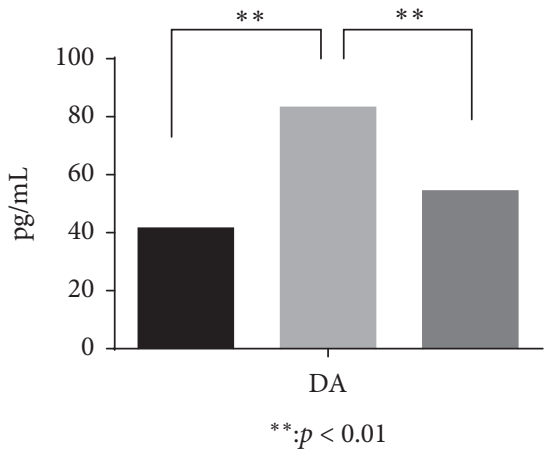

Control group

NTG-induced migraine

DCXF group

(c)

FIgURE 4: The rat plasma of 5-HT, 5-HIAA, CGRP, DA, NO, and NOs values in the control group, model group, and DCXF group $(n=8$, $x \pm s)$.

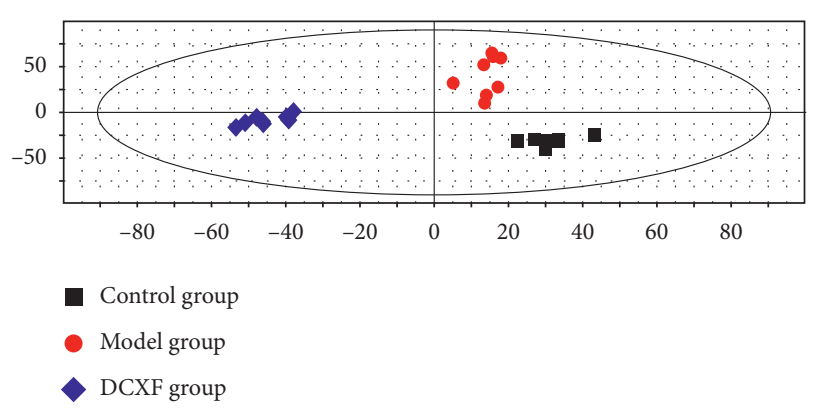

(a)

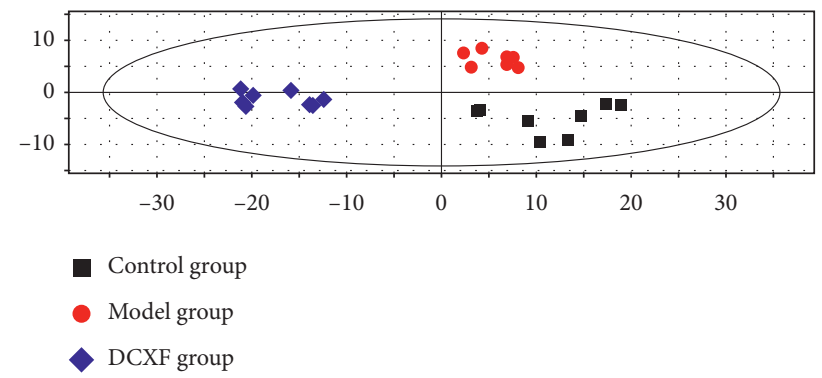

(b)

Figure 5: The PLS-DA clustering score map. (a) Serum at positive ion for control group $(n=8)$, NTG-induced migraine model group $(n=8)$, and DCXF group $(n=8)$; (b) brain tissue at positive ion for the three groups.

TABle 4: Possible pathways for DCXF (FDR: false detection rate).

\begin{tabular}{|c|c|c|c|c|c|}
\hline Serum & FDR & Impact & Brain & FDR & Impact \\
\hline $\begin{array}{l}\text { Alanine, aspartate, and glutamate } \\
\text { metabolism }\end{array}$ & 0.00030 & 0.621 & Aminoacyl-tRNA biosynthesis & $6.85^{*} 10^{-12}$ & 0.167 \\
\hline Arginine biosynthesis & 0.0097 & 0.117 & Valine, leucine and isoleucine biosynthesis & 0.00106 & 0 \\
\hline Butanoate metabolism & 0.0097 & 0.0318 & Alanine, aspartate and glutamate metabolism & 0.0375 & 0.337 \\
\hline Tryptophan metabolism & 0.012 & 0.393 & Arginine biosynthesis & 0.00556 & 0 \\
\hline Tyrosine metabolism & 0.012 & & Pantothenate and CoA biosynthesis & 0.0103 & 0 \\
\hline Nitrogen metabolism & 0.0037 & 0.017 & Glyoxylate and dicarboxylate metabolism & 0.0280 & 0.0423 \\
\hline D-Glutamine and D-glutamate metabolism & 0.0089 & 0.018 & $\begin{array}{c}\text { Phenylalanine, tyrosine and tryptophan } \\
\text { biosynthesis }\end{array}$ & 0.0332 & 0.5 \\
\hline Glyoxylate and dicarboxylate metabolism & 0.016 & 0.042 & D-Glutamine and D-glutamate metabolism & 0.0494 & 0 \\
\hline
\end{tabular}

widely used platform ( $>300,000$ users) dedicated to metabolomics data analysis via a user-friendly and webbased interface [27, 28]. Amino acid metabolism is a common pathway for DCXF in serum and brain tissue. In addition, alanine, aspartate, and glutamate metabolism may be the metabolic pathways of DCXF in both serum and brain tissue. Differences in potential pathways obtained from serum and brain tissue might lead to different mechanisms, which could provide crucial information for understanding the mechanisms associated with DCXF.

3.7. Gene-Metabolite Interaction Network. The Network Explorer module in MetaboAnalyst is an easy-to-use tool 
that permits the mapping of metabolites and/or genes (including KEGG orthologs or KOs) onto different types of molecular interaction networks, as well as molecule-phenotype association networks [27-29]. The gene-metabolite interaction network was enabled for the exploration and visualization of interactions between functionally related metabolites and genes. The human gene associations were extracted from STITCH database, such that only interactions identified with high confidence were used.

To explore the possible gene-metabolite interaction network related to the impact of DCXF, the hub genes and metabolites that contributed to the change of the metabolic state resulting from DCXF were imported to the MetaboAnalyst 4.0. The results are shown in Figure 6. Tables are shown in Supplementary S11.

The results of the gene-metabolite interaction network in serum metabonomics (Figure 6) showed that hub genes were related to DA, Glu, 5-HT, GABA, choline, Gln, Asp, L-Valine, 5-HIAA, L-lactic acid, Try, and vanylglycol. The most relevant metabolites were DA, Glu, and 5-HT with a degree of 15,14 , and 14, respectively, and betweenness of 100.57, 106.82, and 94.71, respectively.

CHAT, COMT, GCG, FOS, and CASP3 were the most relevant genes with metabolites at degrees of 7, 6, 6, 6, and 5, respectively. ADCY2, APP, FOS, IL6, CREB1, ACHE, GCG, CHAT, COMT, and HCRT were linked with DA, Glu, and 5HT.

Figure 6 showed the most relevant metabolites to be AA, Gln, and Asp with degrees of 10, 4, and 3, respectively, and betweenness of 115.1,29.9, and 20, respectively. CASP3 was linked with AA, Gln, and Asp. GCG, CASP3, CHAT, IL6, and JUN also contributed to metabolites associated with brain tissue metabonomics. The GTEx RNA-seq data were used to verify the expression of hub genes in the brain tissues (Supplementary S12). ADCY2, APP, FOS, IL6, CREB1, ACHE, CASP3, and COMT showed the highest expression among the subregions representing the brain tissue.

The most relevant metabolites were Glu and Asp both in serum and brain tissue, and these results coincided with those of the possible metabolic pathway of DCXF (alanine, aspartate, and glutamate metabolism). In addition, the hub genes of DCXF may affect mainly neurotransmitters in serum metabonomics, while in brain tissue metabonomics, the hub genes of DCXF may mostly be linked with inflammation, especially with AA.

\section{Discussion}

In this study, the antimigraine mechanism of DCXF was elucidated using network pharmacology combined with metabolomics techniques. Network pharmacology analysis demonstrated that multiple components of DCXF exerted a therapeutic effect on migraine by modulating multiple targets and pathways. Of these components, the main compounds of DCXF (e.g., SEI, gastrodin, and ferulic acid $[7,8]$ ) were shown to have the potential for migraine treatment, which confirmed the therapeutic value of DCXF against this disease. We found 532 genes as potential targets of DCXF by network-based target prediction. Furthermore,
33 genes associated with both migraine and DCXF were investigated, and most of the intersecting genes were 5hydroxytryptamine receptors, dopamine receptors, and peptide receptors.

DCXF has been found to affect the expression of the 5HT1D receptor, upregulate the expression of monoamine neurotransmitter 5-HT1B receptor, and downregulate the expression of C-JUN [30, 31]. It may also significantly inhibit the expansion of dural blood vessels caused by NTG modeling and the activation of NOS, SP, and CGRP receptors caused by NO [32]. In addition, DCXF induced a downward trend in expression of the dopamine D2 receptor in the midbrain trigeminal nucleus [33].

In our previous study, we focused on the action of DCXF on the peptide receptor. The mechanism of DCXF in migraine management may be associated with its capability in downregulating CGRP expressions in the hypothalamus and PAG, reducing CGRP synthesis, and inhibiting neurogenic inflammation [7]. Combining previous research with the present findings, we hypothesize that various compounds in DCXF may act on migraine through different biological pathways, which may be highly dependent on the 5-hydroxytryptamine receptors, dopamine receptors, and peptide receptors.

Studies have suggested possible endogenous metabolite biomarkers in the development and progression of migraine, including glutamate/glutamine [10], aspartate [10], GABA [34], PC [35], choline [35], lactate [36], and fatty acids [37]. Amino acid metabolism is vital to the understanding of migraine. Amino acids not only consist of units of protein but also play roles in signal transduction and participate in metabolic pathways as neurotransmitters. In addition to affecting amino acid metabolism, prevalent triggers of migraine attacks could all be linked to unbalanced cerebral energy metabolism, especially lactic acidosis (a nonspecific hallmark of mitochondrial disorders). Recent findings have suggested an association between migraine and changes in high-density lipoprotein subspecies (HDL) metabolism rather than general dyslipidaemia [37]. This may suggest that the migraine is linked with lipid metabolism. In our study, the results of GO and KEGG enrichment analysis showed that DCXF significantly regulated the dopamine metabolic process, glucose transmembrane transport, lipid metabolism, fatty acid transport, tyrosine metabolism, and tryptophan metabolism.

From the metabolomics study (Figure 7), the therapeutic effects of DCXF and its possible mechanisms in vivo verified the same antimigraine effect on the changes of endogenous neurotransmitters (5-HT, 5-HIAA, CGRP, DA, NO, and NOs) and similar metabolic (glutamate and aspartate, tyrosine, tryptophan, energy, and lipid metabolism) trends in serum and brain tissue. After NTG modeling, 5-HT and 5HIAA increased significantly in serum.

Tryptophan is metabolized in the brain by tryptophan hydroxylase 2 (TPH2) to produce 5-HTP, and 5-HTP generates 5 -HT via serotonin decarboxylase. 5-HT generates 5-HIAA under the action of monoamine oxidase (Mao) [38-41]. Therefore, 5-HT led tryptophan to be increased in the brain and decreased in the blood. DCXF regulated 


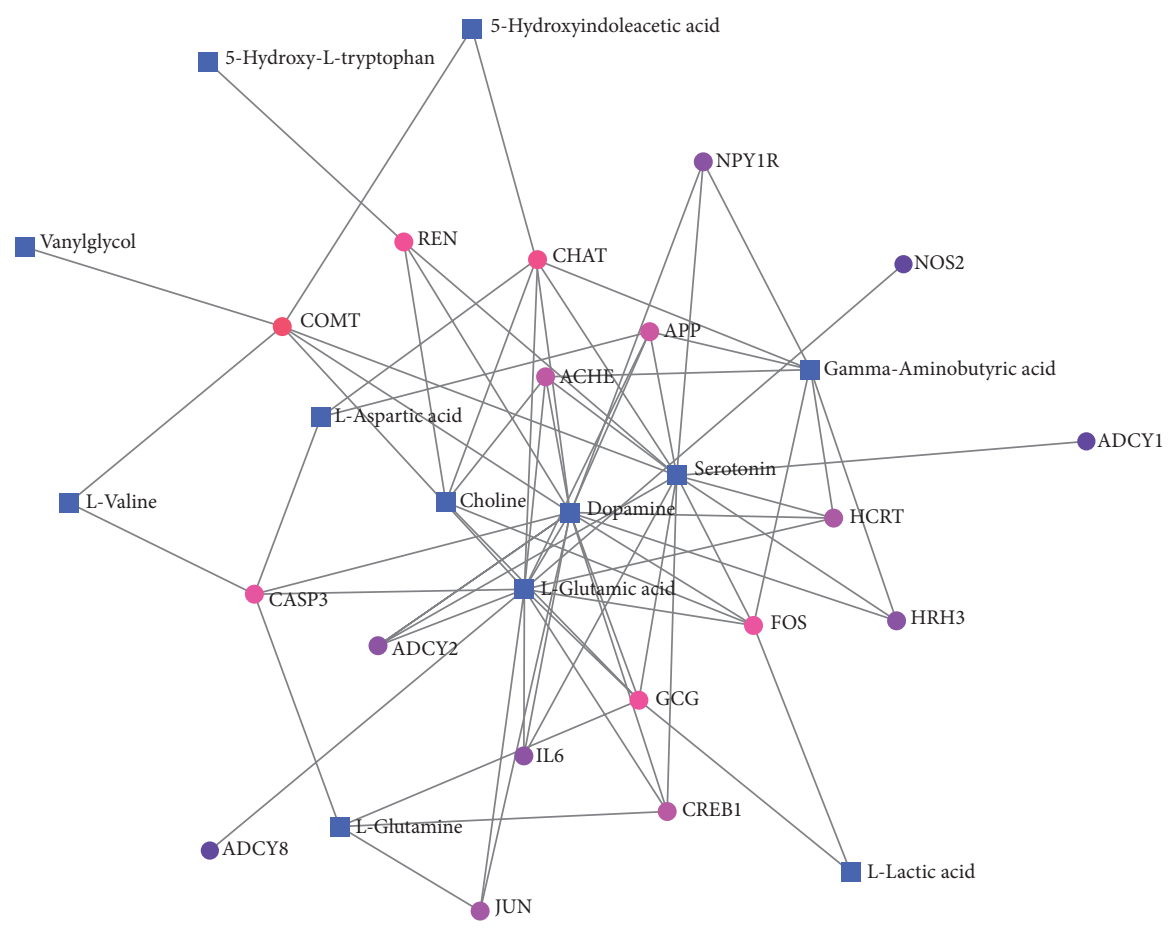

(a)

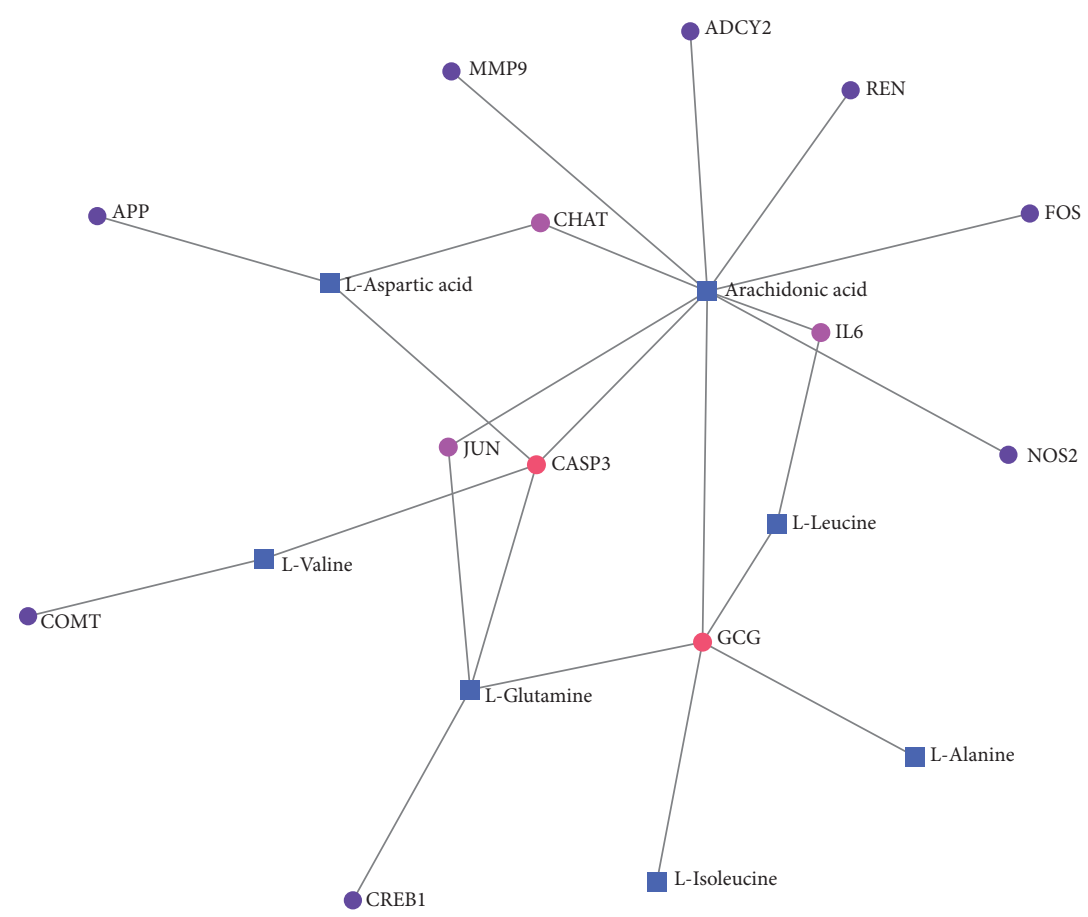

(b)

FIGURE 6: Gene-metabolite interaction network with hub genes and serum metabolites (a) and brain tissue (b) metabolites.

tryptophan metabolism, by increasing it in serum and reducing 5-HT, 5-HIAA, and 5-HTP to further relieve the increased neurotransmitter after NTG modeling. Excitatory amino acids, such as Glu, Gln, and asp, increased significantly in the blood in migraine attacks, while brain norepinephrine levels dropped, causing a dopamine waterfall effect in the sympathetic nerve endings [41]. This forces tyrosine levels to increase in the brain and decrease in the blood, and then, DA, MHPG, and norepinephrine are synthesized in the brain, thus leading to increased blood content. The changes in these transmitters affect the central nervous system of migraine patients, leading to 


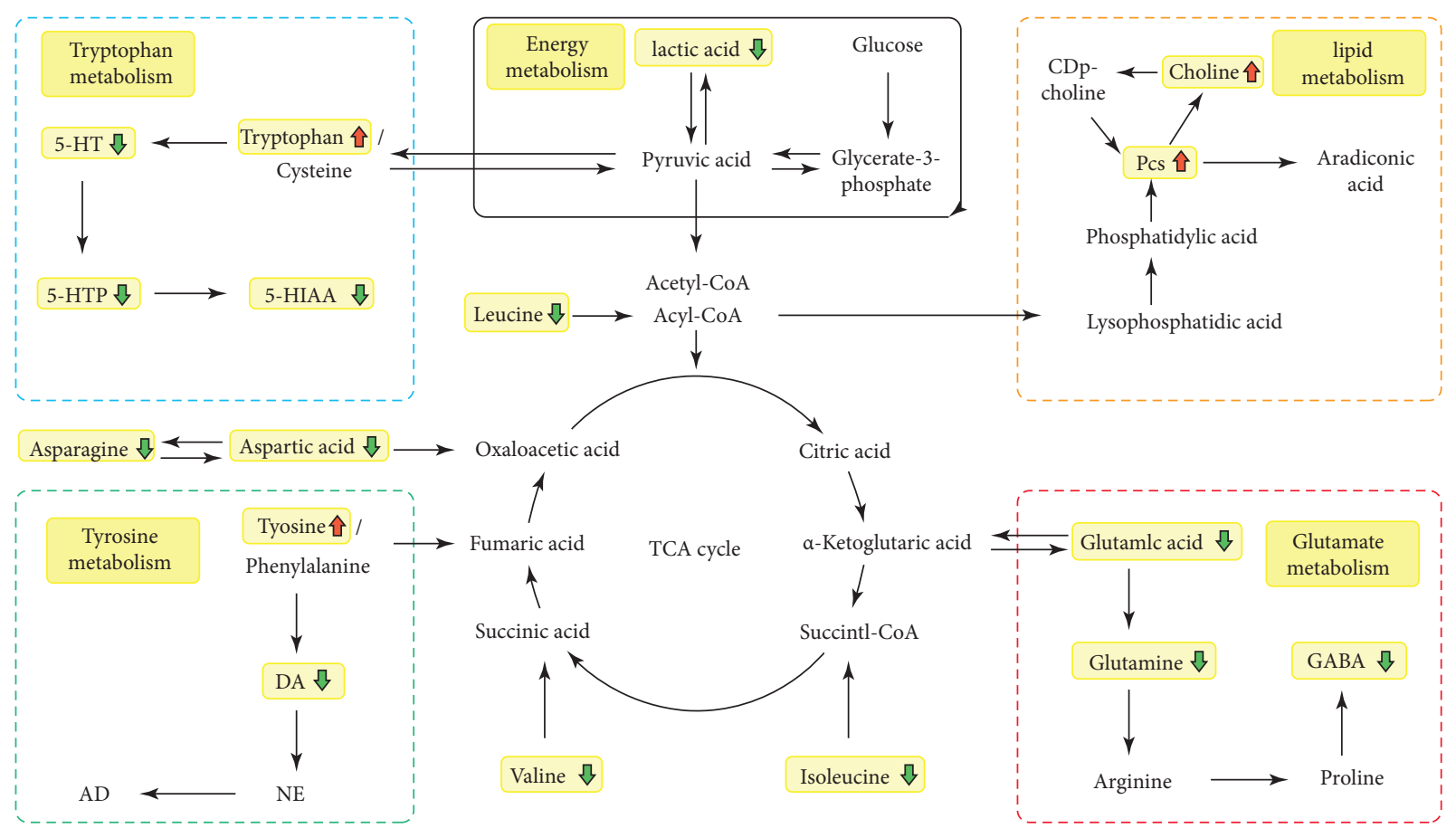

Figure 7: The possible metabolic pathways of DCXF in case of migraine.

mitochondrial dysfunction, increased glutamate levels, and release of CGRP, which contribute to sensitization and inflammation of nerves [42].

DCXF may increase tyrosine and decrease DA. In our previous study, we found that DCXF could improve the metabolic profile of serum and TCC in migraine rats and showed the process of treatment over time, involving mainly amino acid (glutamate and aspartic acid) metabolism. In the present study, in terms of brain tissue metabolomics, Asp and Glu were markedly increased in the NTG-induced migraine model group compared with the control group. DCXF had the same effect on glutamate and aspartic acid metabolism, by decreasing asparagine (synthesized from aspartic acid and ATP), aspartic acid, glutamine acid, glutamine, and GABA (transformed from glutamate catalyzes decarboxylation by glutamate decarboxylase). We also found that DCXF may affect lactic acid, choline, and PCs by regulating energy and lipid metabolism. This was embodied in the increased serum contents of choline and phosphatidylcholine along with decreased lactic acid content in rats, indicating the anaerobic glycolysis of glucose. Anaerobic glycolysis of pyruvic acid would produce pyruvic acid, valine, and leucine and would increase their levels in blood and brain. Most amino acids can be transformed into each other or other types of substances (such as lipids or carbohydrates) through the tricarboxylic acid cycle. The metabolomics approach in the present study found no metabolites closely linked with the TCA cycle. One explanation might be that our study mainly focused on the metabolic trend after 30 minutes of DCXF administration. In our previous study, we found that DCXF regulated succinic acid and citric acid after 90 minutes of administration. Taken together, these findings may suggest that DCXF firstly regulates amino acid metabolism (30 minutes) and later affects energy and lipid metabolism (90 minutes).

Using the aforementioned two technologies in combination, the gene-metabolite interaction networks with hub genes and metabolites (serum and brain tissue) were established. Overall, in serum and brain tissue, the most relevant metabolites were Gln and AA, which were also key to the possible metabolic pathway of DCXF (alanine, aspartate, and glutamate metabolism). Using serum metabolomics, we also found that the hub genes of DCXF may affect mainly neurotransmitters and may mostly be linked with inflammation in brain tissue metabolomics, particularly with AA, which was notably elevated in brain tissue of the model group. This might be attributed to peroxidation of the cell membrane by reactive oxygen species production, thus contributing to the release of the cell phospholipase. The increased serine and alanine in the brain also suggest halted folic acid anabolism or Gln synthesis.

We found that arachidonic acid metabolism was closely related to 5-HT. Previous research [43] has found that arachidonic acid and its metabolites serve as messengers within or between cells. They may oxidize the coupling of 5HT2A (5-HT2B receptor), activate the 5-HT2A/PLA2 signal pathway, and have an effect on migraine. With the establishment of a gene-metabolite interaction network, we found some significant hub genes of DCXF that were associated with metabolites (Figure 8). CASP3 was linked with AA, Gln, and Asp in brain tissue metabolism. The protein encoded by CASP3 is a cysteine-aspartic acid protease that plays a central role in the execution phase of cell apoptosis. In injury, AA activates a stress response resulting in CASP3 activation, which contributes to cerebral vascular damage and dysfunction with an increase in CASP3 levels during 


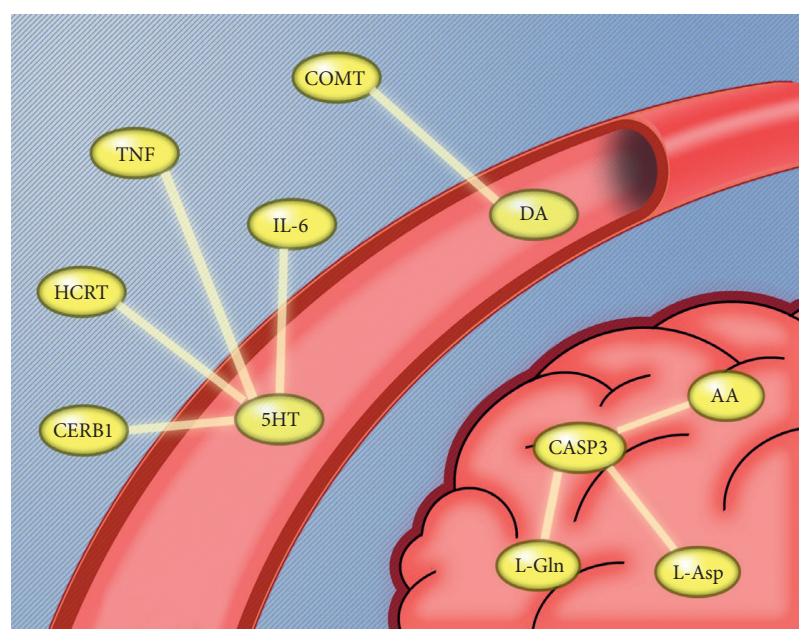

Figure 8: Some of the hub genes linked with metabolites.

neuroinflammation [44]. CASP3 may accelerate neuronal cell death in several cortical spreading depression-related neurological disorders, such as stroke, migraine, and epilepsy [45]. CERB1, HCRT, IL-6, and TNF have been mainly linked with 5-HT. CERB1 encodes a transcription factor, which is a member of the leucine zipper family of DNA binding proteins, and bonds as a homodimer to the cAMPresponsive element, an octameric palindrome. Upon exposure of sensory neurons to the neurotransmitter 5-HT, CREB1 is activated via the protein kinase A (PKA) intracellular signaling pathways [46].

IL-6 and TNF are primarily produced at the sites of acute and chronic inflammation, where they are secreted into the serum and induce a transcriptional inflammatory response. Some studies [45] have underlined that peripheral inflammation (injection with TNF and/or IL-6) reached the hypothalamus, where it affected serotonergic metabolism. A hypothalamic neuropeptide precursor protein encoded by HCRT has been found to give rise to two mature neuropeptides. It was proposed that HCRT may be responsible for migraines, mainly in the early phases of attack, as hypocretinergic neurons originate from the hypothalamus and control the serotonergic system [45]. Another study found that the COMT gene was mainly associated with dopamine, degrading dopamine and its precursor 1-DOPA and also playing a critical role in regulating synaptic dopamine actions [46].

In the present study, MCODE and hub gene analysis of PPI in DCXF found that DCXF could act on inflammationrelated genes, such as IL-10, TNF, IL-6, which were mainly enriched under adenylate cyclase activity and guanylate cyclase activity and in response to forskolin (FSK). Other studies found that active ingredients, such as gastrodin $[47,48]$ and ligustrazine [49], from DCXF reduced the serum levels of proinflammatory cytokines (IL-6, IL- $1 \beta$, and $\mathrm{TNF}$ ), increased the activity of superoxide dismutase, and reduced the concentration of malondialdehyde. In recent studies, high serum levels of IL-1 $\beta$, IL-6, and TNF (proinflammatory cytokines) in patients with migraine indicated that migraine was associated with inflammation within peripheral endings of trigeminal ganglion sensory neurons [50]. So, CGRP, TNF- $\alpha$, and IL- $1 \beta$ were proposed as therapeutic targets of migraine $[3,43]$. It is well known that FSK, as an adenylate cyclase-specific activator, can upregulate intracellular cAMP levels and affect the BBB permeability. It could also evoke an increased NO release from trigeminal satellite glial cells, which given the putative role of $\mathrm{NO}$ in painful conditions such as migraine could be modulated by Glu [43]. Moreover, FSK could potentiate the TNF- $\alpha$ mediated upregulation of BDNF expression. In other studies, LC acts as a vasodilator to increase the levels of cyclic adenosine monophosphate (cAMP) and cyclic guanosine monophosphate (cGMP) in muscle and blood and may be partly mediated by the inhibition of cAMP phosphodiesterase or cGMP phosphodiesterase. Since DCXF consists of both GE and LC, its regulation of cAMP and cGMP might be different from that of LC. The results of the MCODE analysis and the hub genes might provide a new hypothesis of DCXF on antimigraine mechanisms.

\section{Conclusions}

Our results firmly supported and enhanced the current understanding of the therapeutic effects of DCXF on migraine. We have demonstrated an effective strategy combining network pharmacology and metabolomics to understand the mechanisms of action of DCXF. Our findings may facilitate the generation of new hypotheses to reveal the mechanisms of TCM treatment for migraine.

\section{Abbreviations}

DCXF: Da Chuan Xiong Fang

TCM: Traditional Chinese medicine

NSAIDs: Nonsteroidal anti-inflammatory drugs

DHE: Dihydroergotamine

MOH: Medication overuse headache

TCC: Trigeminocervical complex

Trp-KYN: Tryptophan-kynurenine pathway

GABA: g-Aminobutyrate acid

5-HT: $\quad$ Serotonin

BBB: Blood-brain barrier

EAAs: Excitatory amino acids

MMP-9: Matrix metalloprotein-9

PAG: Periaqueductal gray

LC: $\quad$ Ligusticum striatum DC.

GE: Gastrodia elata Blume

CDMT: Codergocrine mesylate tablets

5-HIAA: 5-Hydroxyindolacetic acid

NO: $\quad$ Nitrogen monoxide

NOs: $\quad$ Nitric oxide synthase

CGRP: Calcitonin gene-related peptide

DA: Dopamine

QED: Quantitative estimate of drug-likeness

RMSD: Standard root-mean-square deviation

MF: Molecular function

CC: Cellular component

BP: Biological process

MCODE: Molecular complex detection 
PLS-DA: Partial least square discriminant analysis

VIP: Variable importance in the projection

NTG: Nitroglycerin

Trp: $\quad$ Tryptophan

Tyr: Tyrosine

PC: $\quad$ Phosphatidylcholines

5-HTP: 5-Hydroxytryptophan

Glu: $\quad$ Glutamic acid

Gln: $\quad$ Glutamine

Asp: $\quad$ Aspartic acid

SOD: $\quad$ Superoxide dismutase

MDA: Malondialdehyde

cAMP: Cyclic adenosine monophosphate

cGMP: Cyclic guanosine monophosphate

FSK: $\quad$ Forskolin

AA: $\quad$ Arachidonic acid

PKA: $\quad$ Protein kinase A.

\section{Data Availability}

The datasets used for the current study are available from the corresponding author upon request

\section{Ethical Approval}

The animal facilities and protocols were approved by the Institutional Animal Care and Use Committee, Shanghai University of Traditional Chinese Medicine, on 4th September 2016 (no. SZY201609010). All procedures were conducted according to the Guide for the Care and Use of Laboratory Animal (The National Academies Press, revised edition 2010). All the experimentation was carried out in strict accordance with the established guidelines on the use of experimental animals. All sections of this report adhere to the ARRIVE Guidelines for reporting animal research. A completed ARRIVE guidelines checklist was included in Supplementary S6

\section{Conflicts of Interest}

The authors declare that they have no conflicts of interest.

\section{Authors' Contributions}

Shiyu Ma and Lin Zheng authors contributed equally to this work; they did the experiment, performed the analyses, conducted the literature search, and drafted the manuscript. Lan Shen took part in the design of the study and revised the manuscript. Shiyu Ma and Ming Yang did the network pharmacology study. Lan Shen, Ming Yang, Yi Feng, and Xiao Lin helped interpret findings and critically reviewed the manuscript. All authors read and approved the final manuscript.

\section{Acknowledgments}

The authors would like to thank Dr. Weijie LIN from Zhuhai hospital affiliated with Jinan University for the help during the manuscript modification and revision. The authors also would like to thank the "Charlesworth Author Services" for editing the language of the paper. This work was supported by grants from National Thirteenth Five-Year Science and Technology Major Special Project for New Drug Innovation and Development, The construction of a demonstration technology platform for the clinical evaluation of new drugs for malignant tumor and other diseases (2017ZX09304001), National Thirteenth Five-Year Science and Technology Major Special Project for Infectious Diseases (2017ZX10305501-002), Natural Science Foundation of Shanghai, China (19ZR1457700), Shanghai University of Traditional Chinese Medicine, reserving outstanding TCM talents, Guanghua Hospital "Star of Hope" Education Project (2020100100052), and Shanghai Municipal Commission of Health Family Planning (ZY3-CCCX-3-5001).

\section{Supplementary Materials}

Supplementary S1: preparation, quality control, and HPLC of DCXF, GE, and LC. Supplementary S2: ingredients from LC and GE. Supplementary S3: QED results of GE and LC. Supplementary S4: 531 core targets. Supplementary S5: migraine genes. Supplementary S6: ARRIVE statement for animal experiments. Supplementary S7: metabolites of serum of brain tissue. Supplementary S8: all active ingredients molecular docking results. Supplementary S9: results of MCODE. Supplementary S10: effect of DCXF on serum and brain tissue metabolic profiling. Supplementary S11: genemetabolite interaction network. Supplementary S12: GTEx RNA-seq data to verify the expression of hub genes in the brain tissues. (Supplementary Materials)

\section{References}

[1] R. C. Burch, D. C. Buse, and R. B. Lipton, "Migraine," Neurologic Clinics, vol. 37, no. 4, pp. 631-649, 2019.

[2] A. Kahriman and S. Zhu, "Migraine and tension-type headache," Seminars in Neurology, vol. 38, no. 06, pp. 608-618, 2018.

[3] G. L. Peters, "Migraine overview and summary of current and emerging treatment options," The American Journal of Managed Care, vol. 25, no. 2, pp. S23-S34, 2019.

[4] A. Charles, "The pathophysiology of migraine: implications for clinical management," The Lancet Neurology, vol. 17, no. 2, pp. 174-182, 2018.

[5] Q. Wang, L. Shen, S. Ma et al., "Effects of Ligusticum chuanxiong and Gastrodia elata on blood-brain barrier permeability in migraine rats," Die Pharmazie, vol. 70, no. 6, pp. 421-426, 2015.

[6] Y. L. Hong, Y. Feng, D. S. Xu et al., "Study on extraction and purification of active parts from Da ChuanXiong Fang for treatment of migraine," Journal of Chinese Medica Materia, vol. 6, pp. 721-723, 2007.

[7] Q. Wang, L. Shen, S.-Y. Ma et al., "Determination of the levels of two types of neurotransmitter and the anti-migraine effects of different dose-ratios of Ligusticum chuanxiong and Gastrodia elata," Journal of Food and Drug Analysis, vol. 24, no. 1, pp. 189-198, 2016.

[8] Q. Q. Wang, L. Shen, J. Zhang et al., "Effects of active components extracted from Dachuanxiong Decoction on the 
expressions of CGRP and its receptors in rats with migraine," Chinese Traditional Patent Medicine, vol. 40, pp. 14-20, 2018.

[9] S. Y. Ma, L. Shen, X. Lin et al., "Intervention effect of dachuanxiong Fang multi-component preparation on acute migraine rat model by urine metabolomics," Chinese Journal of Experience and Traditional Medicine Formula, vol. 25, pp. 101-107, 2019.

[10] N. Ni, Q. Wang, X. Lin, Y. Hong, Y. Feng, and L. Shen, "Studies on the mechanism of glutamate metabolism in NTGinduced migraine rats treated with DCXF," Evidence-Based Complementary and Alternative Medicine, vol. 2019, p. 1, 2019.

[11] S. Li, "Mapping ancient remedies: applying a network approach to traditional Chinese medicine," Science, vol. 23, pp. 72-74, 2015.

[12] S. Li, "Exploring traditional Chinese medicine by a novel therapeutic concept of network target," Chinese Journal of Integrative Medicine, vol. 22, no. 9, pp. 647-652, 2016.

[13] Q. Qi, R. Li, H.-Y. Li et al., "Identification of the anti-tumor activity and mechanisms of nuciferine through a network pharmacology approach," Acta Pharmacologica Sinica, vol. 37, no. 7, pp. 963-972, 2016.

[14] S. Zu, T. Chen, and S. Li, "Global optimization-based inference of chemogenomic features from drug-target interactions," Bioinformatics, vol. 31, no. 15, pp. 2523-2529, 2015.

[15] S. Ma, L. Shen, M. Chen et al., "The study of metabonomics combined with diversity of intestinal flora in LDP intervention in kidney-yin deficiency hyperthyroid rats," RSC Advances, vol. 5, no. 71, pp. 57975-57983, 2015.

[16] J. Zuo, X. Wang, Y. Liu et al., "Integrating network pharmacology and metabolomics study on anti-rheumatic mechanisms and antagonistic effects against methotrexateinduced toxicidty of qing-Luo-yin," Frontiers in Pharmacology, vol. 9, p. 1472, 2018.

[17] M. Yang, J. L. Chen, L. W. Xu et al., "Navigating traditional chinese medicine network pharmacology and computational tools," Evidence Based Complementary and Alternative Medicine, vol. 2013, 2013.

[18] Z. Zhang, P. Yi, J. Yang et al., "Integrated network pharmacology analysis and serum metabolomics to reveal the cognitive improvement effect of Bushen Tiansui formula on Alzheimer's disease," Journal of Ethnopharmacology, vol. 249, p. 112371, 2020.

[19] W. Zhang, Y. Chen, H. Jiang et al., "Integrated strategy for accurately screening biomarkers based on metabolomics coupled with network pharmacology," Talanta, vol. 211, p. 120710, 2020.

[20] Y.-L. Hua, Q. Ma, Z.-W. Yuan et al., “A novel approach based on metabolomics coupled with network pharmacology to explain the effect mechanisms of Danggui Buxue Tang in anaemia," Chinese Journal of Natural Medicines, vol. 17, no. 4, pp. 275-290, 2019.

[21] M. Yang, J. Chen, L. Xu et al., “A network pharmacology approach to uncover the molecular mechanisms of herbal formula ban-xia-xie-xin-tang," Evidence-Based Complementary and Alternative Medicine, vol. 2018, p. 1, 2018.

[22] M. C. K. Ng, S. Fong, and S. W. I. Siu, "PSOVina: the hybrid particle swarm optimization algorithm for protein-ligand docking," Journal of Bioinformatics and Computational Biology, vol. 13, no. 3, p. 1541007, 2015.

[23] G. Stelzer, N. Rosen, I. Plaschkes et al., "The GeneCards suite: from gene data mining to disease genome sequence analyses," Curr Protoc Bioinformatics, vol. 54, p. 1, 2016.
[24] C. Tassorelli, R. Greco, D. Wang et al., "Nitroglycerin induces hyperalgesia in rats--a time-course study," European Journal of Pharmacology, vol. 464, no. 2-3, pp. 159-162, 2003.

[25] Y. Zhu, Y. Feng, L. Shen et al., "Effect of metformin on the urinary metabolites of diet-induced-obese mice studied by ultra performance liquid chromatography coupled to time-offlight mass spectrometry (UPLC-TOF/MS)," Journal of Chromatography B, vol. 925, pp. 110-116, 2013.

[26] Y. Zhu, W. Cong, L. Shen et al., "Fecal metabonomic study of a polysaccharide, MDG-1 from Ophiopogon japonicus on diabetic mice based on gas chromatography/time-of-flight mass spectrometry (GC TOF/MS)," Journal of Chromatography B, vol. 10, no. 2, pp. 304-312, 2014.

[27] Z. Pang, J. Chong, S. Li, and J. Xia, "MetaboAnalystR 3.0: toward an optimized workflow for global metabolomics," Metabolites, vol. 10, no. 5, p. 186, 2020.

[28] J. Chong, D. S. Wishart, and J. Xia, "Using MetaboAnalyst 4.0 for comprehensive and integrative metabolomics data analysis," Current Protocols in Bioinformatics, vol. 68, p. e86, 2019.

[29] Q. Yao, Y. Xu, H. Yang, D. Shang et al., "Global prioritization of disease candidate metabolites based on a multi-omics composite network," Science Reports, vol. 5, p. 17201, 2015.

[30] Z.-H. Pu, C. Peng, X.-F. Xie et al., "Alkaloids from the rhizomes of Ligusticum striatum exert antimigraine effects through regulating 5-HT1B receptor and c-Jun," Journal of Ethnopharmacology, vol. 237, pp. 39-46, 2019.

[31] L. Wan, F. Zhi-ping, and Z. Wang, "The effect of DaChuanxiong Fang extract on 5-HT1D receptor expression of neurogila," Journal of Ethnopharmacology, vol. 1, pp. 1-2, 2003.

[32] M. Zhou, K. Yang, and Y. Wang, "Effects of Dachuanxiong Recipe on NOS, SP and CGRP receptors in dural blood vessels and trigeminal nucleus of nitroglycerin migraine model rats," Pharmacology and Clinics of Chinese Materia Medica, vol. 25, no. 6, pp. 9-10, 2009.

[33] J. Cheng, Z. Zhang, H. Zhu et al., "Effect of ChuanxiongGastrodia on the expression of dopamine D2 receptor in the midbrain nucleus of trigeminal nerve in migraine model rats," Pharmacology and Clinics of Chinese Materia Medica, vol. 54, no. 12, pp. 873-875, 2019.

[34] R. Greco, C. Demartini, A. M. Zanaboni et al., "Chronic and intermittent administration of systemic nitroglycerin in the rat induces an increase in the gene expression of CGRP in central areas: potential contribution to pain processing," Journal of Headache Pain, vol. 19, no. 1, p. 51, 2018.

[35] E. C. Gross, M. Lisicki, D. Fischer, P. S. Sándor, and J. Schoenen, "The metabolic face of migraine - from pathophysiology to treatment," Nature Reviews Neurology, vol. 15, no. 11, pp. 627-643, 2019.

[36] S. Cevoli, V. Favoni, and P. Cortelli, "Energy metabolism impairment in migraine," Current Medicinal Chemistry, vol. 26, no. 34, pp. 6253-6260, 2019.

[37] G. L. J. Onderwater, L. Ligthart, M. Bot et al., "Large-scale plasma metabolome analysis reveals alterations in $\mathrm{HDL}$ metabolism in migraine," Neurology, vol. 92, no. 16, pp. e1899-e1911, 2019.

[38] M. D. Ferrari, J. Odink, C. Tapparelli, G. M. J. Van Kempen, E. J. M. Pennings, and G. W. Bruyn, "Serotonin metabolism in migraine," Neurology, vol. 39, no. 9, p. 1239, 1989.

[39] G. D'Andrea, D. D'Amico, G. Bussone et al., "The role of tyrosine metabolism in the pathogenesis of chronic migraine," Cephalalgia: An International Journal of Headache, vol. 33, no. 11, pp. 932-937, 2013. 
[40] C. Tournois, V. Mutel, P. Manivet, J.-M. Launay, and O. Kellermann, "Cross-talk between 5-hydroxytryptamine receptors in a serotonergic cell line," Journal of Biological Chemistry, vol. 273, no. 28, pp. 17498-17503, 1998.

[41] M. Yücel, D. Kotan, G. Gurol Çiftçi, I. H Çiftçi, and H. I Cikriklar, "Serum levels of endocan, claudin-5 and cytokines in migraine," European Review for Medical and Pharmacological Sciences, vol. 20, no. 5, pp. 930-936, 2016.

[42] G. D'andrea, R. Ostuzzi, A. Bolner et al., "Study of tyrosine metabolism in eating disorders. Possible correlation with migraine," Neurological Sciences, vol. 29, no. 1, pp. 88-92, 2008.

[43] J. Evans, Y. Ko, W. Mata et al., "Arachidonic acid induces brain endothelial cell apoptosis via p38-MAPK and intracellular calcium signaling," Microvascular Research, vol. 98, pp. 145-158, 2015.

[44] L. Hao, Z. Yang, and Y. Bi, "Stochasticity and bifurcations in a reduced model with interlinked positive and negative feedback loops of CREB1 and CREB2 stimulated by 5-HT," Mathematical Biosciences, vol. 274, pp. 73-82, 2016.

[45] M. Kowalska, M. Kapelusiak-Pielok, T. Grzelak et al., "The new *G29A and G1222A of HCRTR1, 5-HTTLPR of SLC6A4Polymorphisms and hypocretin-1, serotonin concentrations in migraine patients," Frontiers in Molecular Neuroscience, vol. 11, p. 191, 2018.

[46] O. Solís, J.-R. García-Montes, P. Garcia-Sanz et al., "Human COMT over-expression confers a heightened susceptibility to dyskinesia in mice," Neurobiology of Disease, vol. 102, pp. 133-139, 2017.

[47] S. Wang, Y. Nan, W. Zhu, T. Yang, Y. Tong, and Y. Fan, "Gastrodin improves the neurological score in MCAO rats by inhibiting inflammation and apoptosis, promoting revascularization," International Journal of Clinical and Experimental Pathology, vol. 11, no. 11, pp. 5343-5350, 2018.

[48] L. Chen, X. Liu, H. Wang, and M. Qu, "Gastrodin attenuates pentylenetetrazole-induced seizures by modulating the mitogen-activated protein kinase-associated inflammatory responses in mice," Neuroscience Bulletin, vol. 33, no. 3, pp. 264-272, 2017.

[49] Y. Li, Z. Zhu, T. Zhang, and Y. Zhou, "Ligustrazine attenuates inflammation and oxidative stress in a rat model of arthritis via the Sirt1/NF- $\kappa \mathrm{B}$ and Nrf-2/HO-1 pathways," Archives of Pharmacal Research, vol. 42, no. 9, pp. 824-831, 2019.

[50] A. B. Oliveira, A. L. L. Bachi, R. T. Ribeiro, M. T. Mello, S. Tufik, and M. F. P. Peres, "Unbalanced plasma TNF- $\alpha$ and IL-12/IL-10 profile in women with migraine is associated with psychological and physiological outcomes," Journal of Neuroimmunology, vol. 313, pp. 138-144, 2017. 